\title{
PROJECTIONS ASSOCIATED WITH JACOBI POLYNOMIALS ${ }^{1}$
}

I. I. HIRSCHMAN, JR.

1. Introduction. The present note is concerned with Jacobi polynomials. However in this section we shall, for the sake of notational simplicity, consider only Legendre polynomials. The statements below, which serve to place our problem in its proper setting are well known and are therefore given without proof.

Let $L^{2}$ be the Hilbert space of measurable functions defined on $(-1 \leqq x \leqq 1)$ with inner product

$$
(a, b)=\int_{-1}^{1} a(x) \overline{b(x)} d x
$$

and let $l^{2}$ be the Hilbert space of functions defined on $(n=0,1,2, \cdots)$ with

$$
(\alpha, \beta)=\sum_{n=0}^{\infty} \alpha(n) \overline{\beta(n)} .
$$

Let $^{2}$

$$
\begin{aligned}
D a(x)= & -\left[\left(1-x^{2}\right) a^{\prime}(x)\right]^{\prime}, \\
\Delta \alpha(n)= & \frac{n+1}{2(n+3 / 2)^{1 / 2}(n+1 / 2)^{1 / 2}} \alpha(n+1) \\
& +\frac{n}{2(n+1 / 2)^{1 / 2}(n-1 / 2)^{1 / 2}} \alpha(n-1) .
\end{aligned}
$$

Restricted to the subspace of $L^{2}$ consisting of those functions $a(x)$ such that $a(x)$ is bounded and $D a(x) \in L^{2}$, the operator $D$ is selfadjoint. Its spectrum consists of the points $\lambda=n(n+1), n=0,1$, $2, \cdots$, the eigen function corresponding to $n(n+1)$ being $(n+1 / 2)^{1 / 2} P_{n}(x)$ where $P_{n}(x)$ is the Legendre polynomial of order $n$. The resolution of the identity $G_{\lambda}(-\infty<\lambda<\infty)$ corresponding to $D, D=\int \lambda d G_{\lambda}$, is given by

Received by the editors May 10, 1956 and, in revised form, June 19, 1956.

1 This research was supported by the United States Air Force, through the Office of Scientific Research and Development Command under Contract AF 18(600)-568.

${ }^{2} \alpha(-1)$ is to be interpreted as 0 . 


$$
G_{\lambda} a(x)=\int_{-1}^{1}\left[\sum_{n(n+1)<\lambda}\left(n+\frac{1}{2}\right) P_{n}(x) P_{n}(t)\right] a(t) d t .
$$

It is possible to consider the projections $G_{\lambda}$ in a more general context. Let $L^{p}(1 \leqq p<\infty)$ consist of those measurable functions on $(-1 \leqq x$ $\leqq 1)$ for which $\|a\|_{p}$ is finite where

$$
\|a\|_{p}=\left[\int_{-1}^{1}|a(x)|^{p} d x\right]^{1 / p}
$$

Pollard in some extremely interesting papers $[3 ; 4 ;$ and 5$]$ has shown that

$$
\left\|G_{\lambda} a(x)\right\|_{p} \leqq A(p)\|a(x)\|_{p} \quad(4 / 3<p<4)
$$

where $A(p)$ depends only upon $p$ and not upon $\lambda$. See also Newman and Rudin [2]. The operator $\Delta$ is selfadjoint on $l^{2}$. Its spectrum consists of the points $(-1 \leqq x \leqq 1)$, the formal eigen function corresponding to $x$ being $(n+1 / 2)^{1 / 2} P_{n}(x)$. (Since the spectrum is continuous $(n+1 / 2)^{1 / 2} P_{n}(x)$ does not belong to $l^{2}$ as a function of $\left.n\right)$. If

$$
\Gamma_{\lambda} \alpha(n)=\sum_{m=0}^{\infty} \alpha(m) \int_{-1}^{\lambda}\left(n+\frac{1}{2}\right)^{1 / 2}\left(m+\frac{1}{2}\right)^{1 / 2} P_{m}(x) P_{n}(x) d x
$$

then $\Gamma_{\lambda}$ is a resolution of the identity and $\Delta=\int \lambda d \Gamma_{\lambda}$. Let $l^{p}$ consist of those functions on $(n=0,1,2, \cdots)$ for which

$$
\|\alpha\|_{p}=\left(\sum_{n=0}^{\infty}|\alpha(n)|^{p}\right)^{1 / p}
$$

In the present note we shall show that

$$
\left\|\Gamma_{\lambda} \alpha(n)\right\|_{p} \leqq B(p)\|\alpha\|_{p} \quad(1<p<\infty),
$$

where $B(p)$ depends only upon $p$ and not upon $\lambda$. This is of course the analogue of Pollard's theorem.

The Legendre polynomials $P_{n}(x)$ have as we have noted a double structure: as functions of $x$ they are eigen functions of a second order selfadjoint differential operator and as functions of $n$ they are eigenfunctions of a second order selfadjoint difference operator. It is the interrelation of these structures which makes possible the demonstration of (4) and (6). The initial step in Pollard's demonstration is to simplify (3) using the difference equation satisfied by $P_{n}(x)$, while here the initial step is to use the differential equation to reduce (5) to a more manageable form. 
2. The main theorem. Let $\sigma \geqq-1 / 2, \tau \geqq-1 / 2$ be fixed. Let

$$
\begin{gathered}
\Omega_{\sigma, \tau}(x)=(1-x)^{\sigma}(1+x)^{\tau}, \\
(2 n+\sigma+\tau+1) n ! \Gamma(n+\sigma+\tau+1) h_{n}^{(\sigma, \tau)} \\
=2^{\sigma+\tau+1} \Gamma(n+\sigma+1) \Gamma(n+\tau+1) .
\end{gathered}
$$

We then have (all formulas such as the one below are taken from [ 1 , vol. 2, Chapter X])

$$
\int_{-1}^{1} P_{n}^{(\sigma, \tau)}(x) P_{m}^{(\sigma, \tau)}(x) \Omega_{\sigma, \tau}(x) d x= \begin{cases}h_{m}^{(\sigma, \tau)} & m=n, \\ 0 & m \neq n .\end{cases}
$$

Let

(1) $\Gamma_{\lambda} \alpha(n)=\sum_{m=0}^{\infty} \alpha(m)\left[h_{n}^{(\sigma, \tau)} h_{m}^{(\sigma, \tau)}\right]^{-1 / 2} \int_{-1}^{\lambda} P_{n}^{(\sigma, \tau)}(x) P_{m}^{(\sigma, \tau)}(x) \Omega_{\sigma, \tau}(x) d x$.

Note that $\Gamma_{\lambda}$ depends upon $\sigma$ and $\tau$ as well as upon $\lambda$.

ThEOREM 1. If $\sigma \geqq-1 / 2, \tau \geqq-1 / 2$ then

$$
\left\|\Gamma_{\lambda} \alpha(n)\right\|_{p} \leqq A(\sigma, \tau, p)\|\alpha(n)\|_{p} \quad(1<p<\infty) .
$$

In what follows we shall exclude the case $\sigma=-1 / 2, \tau=-1 / 2$. Our theorem is true for this case but the formulas need slight changes, which we leave to the reader. If $y_{n}(x)=P_{n}^{(\sigma, \tau)}(x)$ then

$$
\left[\left(1-x^{2}\right) \Omega(x) y_{n}^{\prime}(x)\right]^{\prime}+\Omega(x) n(n+\sigma+\tau+1) y_{n}(x)=0 .
$$

Thus

$$
\begin{aligned}
& n(n+\sigma+\tau+1) \int_{-1}^{\lambda} \Omega(x) y_{n}(x) y_{m}(x) d x \\
& =-\int_{-1}^{\lambda} y_{m}(x)\left[\left(1-x^{2}\right) \Omega(x) y_{n}^{\prime}(x)\right]^{\prime} d x \\
& =-\left[y_{m}(x)\left(1-x^{2}\right) \Omega(x) y_{n}^{\prime}(x)\right]_{-1}^{\lambda}+\int_{-1}^{\lambda} y_{m}^{\prime}(x) y_{n}^{\prime}(x)\left(1-x^{2}\right) \Omega(x) d x, \\
& m(m+\sigma+\tau+1) \int_{-1}^{\lambda} \Omega(x) y_{n}(x) y_{m}(x) d x \\
& =-\int_{-1}^{\lambda} y_{n}(x)\left[\left(1-x^{2}\right) \Omega(x) y_{m}^{\prime}(x)\right]^{\prime} d x \\
& =-\left[y_{n}(x)\left(1-x^{2}\right) \Omega(x) y_{m}^{\prime}(x)\right]_{-1}^{\lambda}+\int_{-1}^{\lambda} y_{n}^{\prime}(x) y_{m}^{\prime}(x)\left(1-x^{2}\right) \Omega(x) d x .
\end{aligned}
$$


Combining these we obtain for $n \neq m$ the equation

$$
\int_{-1}^{\lambda} \Omega(x) y_{n}(x) y_{m}(x) d x=\frac{\left(1-\lambda^{2}\right) \Omega(\lambda)\left[y_{n}(\lambda) y_{m}{ }^{\prime}(\lambda)-y_{m}(\lambda) y_{n}^{\prime}(\lambda)\right]}{(n-m)(n+m+\sigma+\tau+1)}
$$

which is the analogue of the Christoffel-Darboux formula. Let

$$
\begin{aligned}
& \Gamma_{1} \alpha(n)=\sum_{m=0, m \neq n}^{\infty} \alpha(m) \frac{\left(1-\lambda^{2}\right) \Omega(\lambda) y_{n}(\lambda) y_{m}^{\prime}(\lambda) h_{n}^{-1 / 2} h_{m}^{-1 / 2}}{(n-m)(n+m+\sigma+\tau+1)}, \\
& \Gamma_{2} \alpha(n)=\sum_{m=0, m \neq n}^{\infty} \alpha(m) \frac{\left(1-\lambda^{2}\right) \Omega(\lambda) y_{n}^{\prime}(\lambda) y_{m}(\lambda) h_{n}^{-1 / 2} h_{m}^{-1 / 2}}{(n-m)(n+m+\sigma+\tau+1)}, \\
& \Gamma_{3} \alpha(n)=\alpha(n) h_{n}^{-1} \int_{-1}^{\lambda}\left[y_{n}(x)\right]^{2} \Omega(x) d x .
\end{aligned}
$$

Here to simplify the notation we have written $\Omega$ for $\Omega_{\sigma, \tau}$ and $h_{n}$ for $h_{n}^{(\sigma, r)}$. Since $\Gamma_{\lambda}=\Gamma_{1}-\Gamma_{2}+\Gamma_{3}$ it is sufficient to show that $\Gamma_{1}, \Gamma_{2}$, and $\Gamma_{3}$ are bounded linear transformations of $l^{p}$, the bounds being independent of $\lambda$. This is evident for $\Gamma_{3}$. The following inequality is from Szegö $[6$, p. 165].

$$
\left|h_{n}^{-1 / 2}\left(1-\lambda^{2}\right)^{1 / 4} \Omega(\lambda)^{1 / 2} y_{n}(\lambda)\right| \leqq M \quad(-1 / 2 \leqq \sigma,-1 / 2 \leqq \tau) .
$$

Here $M$ depends only upon $\sigma$ and $\tau$ and not upon $\lambda$ or $n$. Since

$$
2 \frac{d}{d x} P_{n}^{(\sigma, \tau)}(x)=(n+\sigma+\tau+1) P_{n-1}^{(\sigma+1, \tau+1)}(x)
$$

we find that

$$
2\left|h_{n}^{-1 / 2}\left(1-\lambda^{2}\right)^{3 / 4} \Omega(\lambda)^{1 / 2} y_{n}^{\prime}(\lambda)\right| \leqq M(n+\sigma+\tau+1) .
$$

Let

$$
\begin{aligned}
& A(n, \lambda)=h_{n}^{-1 / 2}\left(1-\lambda^{2}\right)^{1 / 4} \Omega(\lambda)^{1 / 2} y_{n}(\lambda) \\
& B(n, \lambda)=h_{n}^{-1 / 2}\left(1-\lambda^{2}\right)^{3 / 4} \Omega(\lambda)^{1 / 2} y_{n}^{\prime}(\lambda)\left[n+\frac{1}{2}(\sigma+\tau+1)\right]^{-1}
\end{aligned}
$$

then

$$
\begin{aligned}
|A(n, \lambda)| \leqq M_{1}(\sigma, \tau), \quad & |B(n, \lambda)| \leqq M_{1}(\sigma, \tau) \\
& (n=0,1,2, \cdots ;-1 \leqq \lambda \leqq 1) .
\end{aligned}
$$

If we define 


$$
\begin{aligned}
& T_{1} \alpha(n)=\sum_{m=0, m \neq n}^{\infty} \alpha(m) \frac{m+(\sigma+\tau+1) / 2}{(n-m)(n+m+\sigma+\tau+1)}, \\
& T_{2} \alpha(n)=\sum_{m=0, m \neq n}^{\infty} \alpha(m) \frac{n+(\sigma+\tau+1) / 2}{(n-m)(n+m+\sigma+\tau+1)},
\end{aligned}
$$

then

$$
\begin{aligned}
& \Gamma_{1} \alpha(n)=A(n, \lambda) T_{1}\{\alpha(n) B(n, \lambda)\}, \\
& \Gamma_{2} \alpha(n)=B(n, \lambda) T_{2}\{\alpha(n) A(n, \lambda)\},
\end{aligned}
$$

so that it is sufficient to prove that $T_{1}$ and $T_{2}$ are bounded linear transformations of $l^{p}$. Now

$$
\begin{aligned}
& \left(T_{1}+T_{2}\right) \alpha(n)=\sum_{m=0, m \neq n}^{\infty} \alpha(m) \frac{1}{n-m}, \\
& \left(T_{2}-T_{1}\right) \alpha(n)=\sum_{m=0, m \neq n}^{\infty} \alpha(m) \frac{1}{n+m+\sigma+\tau+1} .
\end{aligned}
$$

It is well known that these transformations of $l^{p}$ are bounded and from this follows the desired result for $T_{1}$ and $T_{2}$; see [7, paragraph 314 .

The same methods suffice to prove that if

$$
\|\alpha(n)\|_{r, p}=\left[\sum_{n=0}^{\infty}|\alpha(n)|^{p}(n+1)^{r p}\right]^{1 / p}
$$

then

$$
\left\|\Gamma_{\lambda} \alpha\right\|_{r, p} \leqq B\|\alpha\|_{r, p} \quad(1<p<\infty ;-1 / p<r<1-1 / p) .
$$

\section{REFERENCES}

1. A. Erdélyi et al., Higher transcendental functions, New York, 1953.

2. J. Newman and Walter Rudin, Mean convergence of orthogonal series, Proc. Amer. Math. Soc. vol. 3, (1952) pp. 219-222.

3. H. Pollard, The mean convergence of orthogonal series, I, Trans. Amer. Math. Soc. vol. 62 (1947) pp. 387-403.

4. - The mean convergence of orthogonal series, II, Trans. Amer. Math. Soc. vol. 63 (1948) pp. 355-367.

5. - The mean convergence of orthogonal series; III, Duke Math. J. vol. 16 (1949) pp. 189-191.

6. G. Szegö, Orthogonal polynomials, New York, 1939.

7. G. H. Hardy, J. E. Littlewood, and G. P6lya, Inequalities, Cambridge, 1934. 